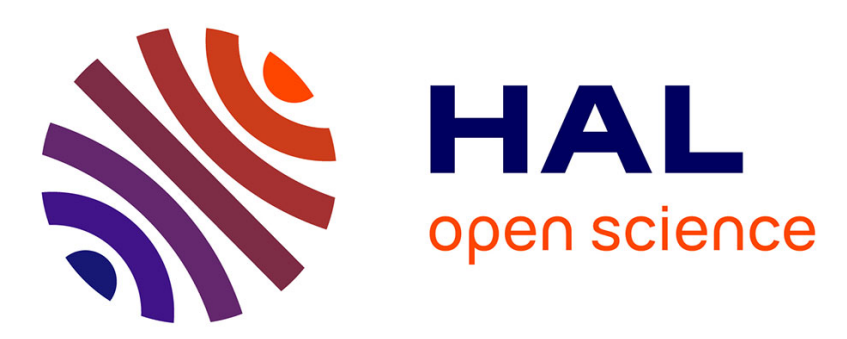

\title{
Bio-Inspired Face Authentication using Multiscale LBP
}

Ayoub Elghanaoui, Nefissa Khiari Hili, Christophe Montagne, Sylvie Lelandais

\section{To cite this version:}

Ayoub Elghanaoui, Nefissa Khiari Hili, Christophe Montagne, Sylvie Lelandais. Bio-Inspired Face Authentication using Multiscale LBP. 6th International Conference on Bio-Inspired Systems and Signal Processing (BIOSIGNAL 2013), Feb 2013, Barcelona, Spain. pp.182-188. hal-00762838

\section{HAL Id: hal-00762838 \\ https://hal.science/hal-00762838}

Submitted on 1 Feb 2014

HAL is a multi-disciplinary open access archive for the deposit and dissemination of scientific research documents, whether they are published or not. The documents may come from teaching and research institutions in France or abroad, or from public or private research centers.
L'archive ouverte pluridisciplinaire $\mathbf{H A L}$, est destinée au dépôt et à la diffusion de documents scientifiques de niveau recherche, publiés ou non, émanant des établissements d'enseignement et de recherche français ou étrangers, des laboratoires publics ou privés. 


\title{
Bio-Inspired Face Authentication using Multiscale LBP
}

\author{
Ayoub Elghanaoui ${ }^{1}$, Nefissa Khiari Hili ${ }^{1,2}$, Christophe Montagne ${ }^{1}$ and Sylvie Lelandais ${ }^{1}$ \\ ${ }^{1}$ IBISC Laboratory, University of Evry Val d'Essonne, 40 Rue du Pelvoux, 91020, Evry Cedex, France \\ ${ }^{2}$ TSIRF Laboratory, ENIT, University of Tunis El Manar, BP-37 Le Belvédère, 1002, Tunis, Tunisia \\ Faouzi-ayoub.elghanaoui@ens.univ-evry.fr,nefissa.khiari-hili@ibisc.univ-evry.fr, \\ cmontagne@iup.univ-evry.fr, s.lelandais@iut.univ-evry.fr
}

\begin{abstract}
Keywords: Face recognition, Local Binary Pattern, Bio Inspired processing, Difference Of Gaussian decomposition.
Abstract: In this paper, we propose a new approach to recognize 2D faces. This approach is based on experiments performed in the field of cognitive science to understand how people recognize a face. To extract features, the image is first decomposed on a base of wavelets using four-level Difference Of Gaussians (DOGs) functions which are a good modeling of human visual system; then different Regions Of Interest (ROIs) are selected on each scale, related to the cognitive method we refer to. After that, Local Binary Patterns (LBP) histograms are computed on each block of the ROIs and concatenated to form the final feature vector. Matching is performed by means of a weighted distance. Weighting coefficients are chosen based on results of psychovisual experiments in which the task assigned to observers was to recognize people. Proposed approach was tested on $\mathrm{IV}^{2}$ database and experimental results prove its efficiency when compared to classical face recognition algorithms.
\end{abstract}

\section{INTRODUCTION}

Human face recognition remains one of the most active areas in security and surveillance applications since it is non-invasive and requires less user cooperation. Most classical approaches for face recognition are holistic appearance-based ones such as Eigenfaces and Fisherfaces (Belhumeur, 1997). On another hand, local feature-based approaches, like Gabor (Li \& Jain, 2005) are believed to achieve high accuracy. Both of them perform well in controlled environments; however, their performances drastically drop when variability like quality, pose and illumination occur. Therefore, new solutions are being suggested to overcome these challenges. Many of them were based on combining conventional algorithms and brought quite good results (Mellakh, 2009; Zhang \& Jia, 2005; Su 2009). Since around 2005 , a lot of studies in the field of face recognition used successfully the "Local Binary Patterns (LBP)" (Huang, 2011).

Now, results of face recognition algorithms are almost around the same values. Improving these results is the goal of new methods. So we propose to explore the work of some psychologists to help the development of automatic algorithm based on textural and multispectral analysis.
The rest of the paper is organized as follows. In a first part we present the bio-inspired work on which we were based to build our method. Then we give some indications about the data and the evaluation protocol we use to evaluate the proposed algorithm. The third part describes the proposed bio-inspired face authentication. Experimentations and comparative results are reported in the fourth part. Finally, we conclude and give some ideas for future works.

\section{WHY A BIO-INSPIRED APPROACH?}

Recently Sinha et al. proposed to take into account the knowledge about the ways people recognize each other (Sinha, 2006). They detailed nineteen important results regarding face recognition by human. In former studies, Gosselin and Schyns proposed a bio-inspired technique called "Bubbles" to reveal the use of information in recognition tasks (Gosselin 2001). To this end, they run a set of experiments on participants (human observers) that had to identify or categorize a set of faces based only on randomly revealed portions of these face images. 
To build the stimuli presented to the observers, Gosselin et al. started by using a Laplacien Pyramid that decomposes an image into six frequency bands in the Fourier domain leading to six scales in the spatial domain (figure 1b). Revealed portions of faces were then obtained by randomly sampling the filtered images with gaussian functions, called Bubbles. After all the observers have answered to the stimuli that were present during the experiment, it was possible to analyze where the information leading observers to give a correct answer was. Figure 1c shows, for each scale, these areas. Then in figure $1 \mathrm{~d}$, we see what parts of the face have been useful, at each scale, for the recognition task. Figure 1e is a reconstruction of the five scales of figure $1 \mathrm{~d}$.

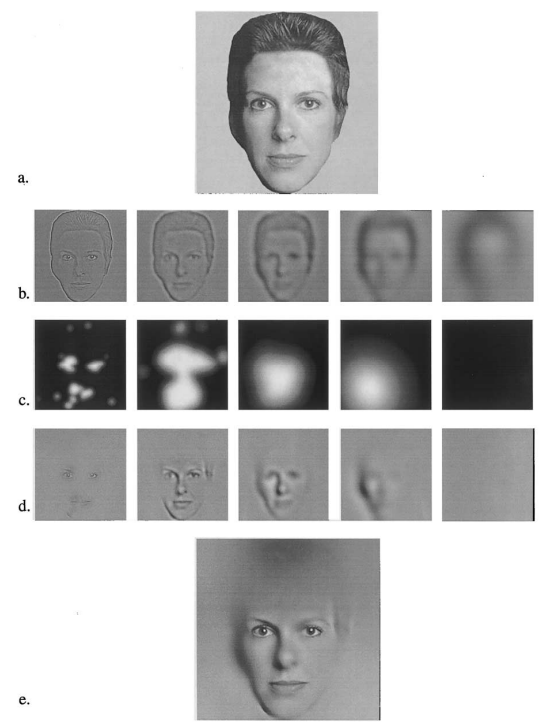

Figure 1: Application of Bubbles; a) Initial image; b) Five different scales of a) ; c) Bubbles applied to each scale; d) Revealed information of b) by the bubbles of c). e) Stimulus resulting by integrating pictures in d) (Gosselin 2001)

To revel which frequency bands were the most discriminative for face recognition (achieving at least $75 \%$ of successful recognition by observers), Schyns et al. (Schyns, 2002) run a study on the proportions of face area that was efficient in each of the five frequency bands already sampled by Bubbles (Figure 2). Results demonstrated that the third scale was the most discriminative followed narrowly by the fourth one. It also has been noticed that the fifth scale did not contribute in any of the identification nor the categorization tasks.

Furthermore, experiment results confirmed that most of the face regions were important for recognition. Specifically, the region of eyes was the most important one as it is present in all scales, followed by the mouth then the nose.

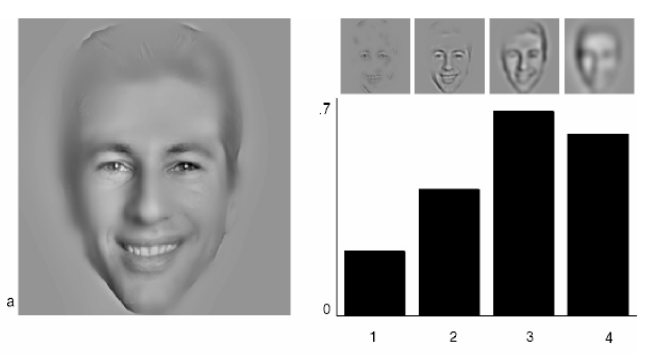

Figure 2: Left: Significant regions for recognition task. Right: bars indicate the proportion of significant pixels in the four first scales (Schyns 2002).

\section{THE IV ${ }^{2}$ DATABASE AND THE EVALUATION PROTOCOL}

In biometric studies, it is very crucial to have a big set of data on which the efficiency of proposed algorithms can be evaluated. Some databases are available but they don't offer enough data either in number or in variability. The IV $^{2}$ database was designed with the aim of proposing multiple test situations to allow evaluation with regard to variability well known to be critical for the biometric systems performance, such as pose, expression, illumination and quality (Figure 3 ). The IV ${ }^{2}$ database has been realized during the Techno Vision program and has been supported by the French Research Ministry in collaboration with the French Ministry of Defense.

The publicly available IV $^{2}$ database (http://lsc.univ-evry.fr/techno/iv2/PageWeb-

IV2.html) allows monomodal and multimodal experiments using face data $(2 \mathrm{D}$ and $3 \mathrm{D}$ face images, 2D stereoscopic face images and infrared iris images). It contains 315 subjects with one session data where 77 of them also participated to a second session. From this database, a subset of 52 subjects, distributed as a development set, constitutes also the training set.

As a closing stage of the $\mathrm{IV}^{2}$ project, an evaluation campaign was performed involving iris recognition, $2 \mathrm{D}$ and $3 \mathrm{D}$-face recognition and also multimodal recognition. In the 2D-Face evaluation (Petrovska, 2008), the strategy of having "one variability" at a time was adopted in order to evaluate how challenging variability - related to illumination, expression, quality or multi-session images - can be for the biometric systems.

In this evaluation campaign a set of more than 15000 images were tested through four experiments. The first three experiments are monosession (all 
images were collected in a unique session). Experiment 1 includes neutral faces and small expression variations. Experiment 2 tests illumination variations and Experiment 3 tests quality variation. Whereas Experiment 4 includes multi-session images, that were collected in three different sessions.

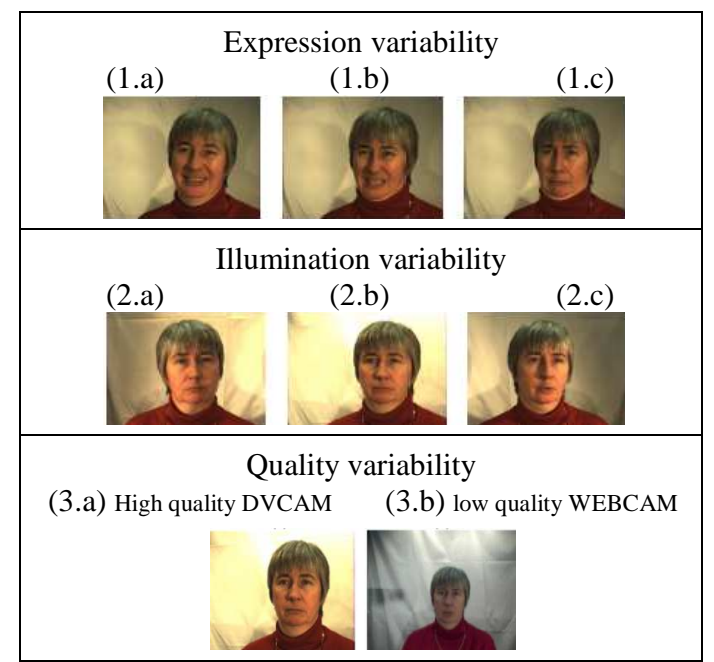

Figure.3. Examples of variability related to (1.a-c) expression, (2.a-c) illumination and (3.a-b) quality.

Five appearance based methods were evaluated on the $\mathrm{IV}^{2}$ database. Details about the algorithms are given in (Mellakh, 2009) and comparative results are shown in Table 4.

\section{PROPOSED METHOD}

The proposed method is based on four steps. First, wavelet decomposition is performed. Then, Regions Of Interest (ROIs) are selected on each scale, related to Gosselin's analysis (Gosselin, 2001). Then LBP operator is computed on each filtered image. Finally, matching is performed by computing a weighted distance between request and stored images. Weighting coefficients were set according to the importance of scales and areas. In the next, theoretical background is presented before detailing the proposed approach.

\subsection{Difference Of Gaussians (DOG)}

Rodieck and Stone showed that the responses of the retinal ganglion cells could be modeled by a Difference Of Gaussians function (DOGs) (Rodieck $\&$ Stone, 1965. To go in the same direction and, at the same time, explore Gosselin and Schyns results we used a DOGs filter instead of Laplacien Pyramid. The DOG's filter formula in image plane is given by (1) :

$$
\operatorname{DOG}(x, y)=C_{1} \exp ^{-\frac{x^{2}+y^{2}}{2 \pi a^{2}}}-C_{2} \exp ^{-\frac{x^{2}+y^{2}}{2 \pi \sigma^{2} a^{2}}}
$$

with $C_{1}, C_{2}, \sigma$ and $a$ are fixed constants following psycho-visual experiments $\left(C_{1}=1.8, C_{2}=0.8, \sigma^{2}=2.25\right)$ as shown by Schor et al. (Schor 1983); $a$ is the scale of the DOG.

\subsection{Local Binary Patterns (LBP)}

The original LBP operator was first used in texture analysis in 1999 (Pietikäinen, 1999). It is a simple yet effective non parametric descriptor that labels the pixels of an image by thresholding a $3 \times 3$ neighborhood of each pixel with the center value and considering the results as a binary number called Local Binary Pattern (see figure 4).

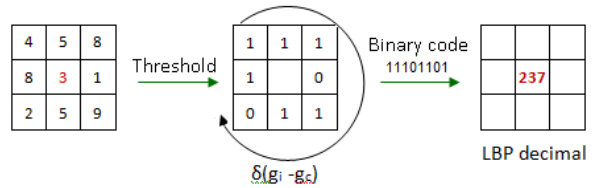

Figure 4: An example of LBP operator $(\mathrm{P}=8, \mathrm{R}=1)$.

Ojala et al. later made an extension of the original operator to allow any radius and any number of pixels in the neighborhood (Ojala, 2002). The notation $L B P_{P, R}$ denotes an LBP with a neighborhood of $P$ equally spaced sampling points on a circle of radius $R$. It can be expressed in decimal form for a given pixel at $\left(x_{c}, y_{c}\right)$ as:

$$
L B P_{P, R}\left(x_{c}, y_{c}\right)=\sum_{i=0}^{P-1} 2^{i} \delta\left(g_{i}-g_{c}\right)
$$

where $i$ runs over the $P$ neighbours of the central pixel, $g_{c}$ and $g_{i}$ are the gray-level values of the central pixel and the surrounding pixel. $g_{i}$ is of coordinates $\left((-R \sin (2 \pi i / P), R \cos (2 \pi i / P))\right.$ if the coordinates of $g_{c}$ are $(0,0) . \delta(x)$ is 1 if $x>0$ and 0 otherwise. Figure 4 shows an example of an original $\mathrm{LBP}_{8,1}$.

After labeling an image with an LBP operator, the histogram of the labeled image is computed giving an LBP operator which can be exploited as a texture descriptor. The $L B P_{P, R}$ produces $2^{P}$ different binary patterns that can be formed by the $P$ pixels in the neighbor set. This leads to 256 patterns for $\operatorname{LBP}_{8,1}$.

Some variations of the original LBP have been developped. The most known of them are the rotation invariance $L B P^{R I}$ (Pietikäinen, 2000), the uniform pattern $\mathrm{LBP}^{\mathrm{U} 2}$ (Mäenpää, 2000) and the rotation invariant uniform pattern $\mathrm{LBP}^{\mathrm{RIU} 2}$ (Ojala, 2002). Recent studies (Huang, 2011) demonstrated that more than $90 \%$ of the discriminative patterns for representing faces were uniform. 


\subsection{Chi-Square distance $(\chi 2)$}

To measure similarity between two LBP histograms $H_{1}$ and $H_{2}$ of two images $I_{1}$ and $I_{2}$, we use Chi-Square distance $\chi^{2}$ that has been widely used in face recognition (Huang, 2011), given by the following formula:

$$
\chi^{2}\left(H_{1}, H_{2}\right)=\sum_{i} \frac{\left(H_{1 i}-H_{2 i}\right)^{2}}{\left(H_{1 i}+H_{2 i}\right)}
$$

$i$ is the elements' index of $H_{1}$ and $H_{2}$.

\subsection{Implementation}

Here are given the different steps of the proposed method, starting by feature extraction from $128 \times 128$ normalized face images through DOG filtering, then LBP calculation on ROIs subdivided into blocks and finally ending by matching by means of weighted Chi-square distance.

\subsubsection{DOG filtering}

In this work, wavelets are performed through a direct calculation in Fourier plane using the analytic formula of the DOG (Eq. 1): each image is decomposed at the input of the algorithm into four frequency bands, each containing one octave. Figure $5 \mathrm{~b}$ presents the results of such a decomposition performed on the original image $5 \mathrm{a}$. The four scales used are equal to $1,2,4$ and 8 .

\subsubsection{Regions of interest selection (ROIs)}

The first application of the bio-inspired approach in the proposed method consists in focusing only on the discriminant regions of each scale, based on the results found by Gosselin et al. (Gosselin, 2001).

In fact, their studies state that for the first scale, participants focused only on the eyes and mouth regions; for the second scale, they added the nose; while for the third and fourth scales they used all the face regions in the recognition task. The ROIs selection is illustrated in Figure 5c.

\subsubsection{LBP application on ROIs}

Once the regions are chosen, they are labeled with an LBP operator then subdivided into non overlapping sub-blocks. Histograms of the labeled blocks are then calculated and concatenated into a single histogram constituting the final feature vector. This representation allows capturing both local texture (LBP, sub-block division) and global shape (histogram concatenation) of face. Figure $5 \mathrm{~d}$ shows an example of a $128 \times 128$ face image where the ROIs of each scale are subdivided into $16 \times 16$ blocks leading to a total of 182 blocks where LBP histograms (of size 59 in case of $\mathrm{LBP}^{\mathrm{U} 2}$ ) are computed then concatenated into a single feature vector (of size 10738 in case of $\mathrm{LBP}^{\mathrm{U} 2}$ ) as illustrated in Figure 5e.

$$
\text { a) }
$$
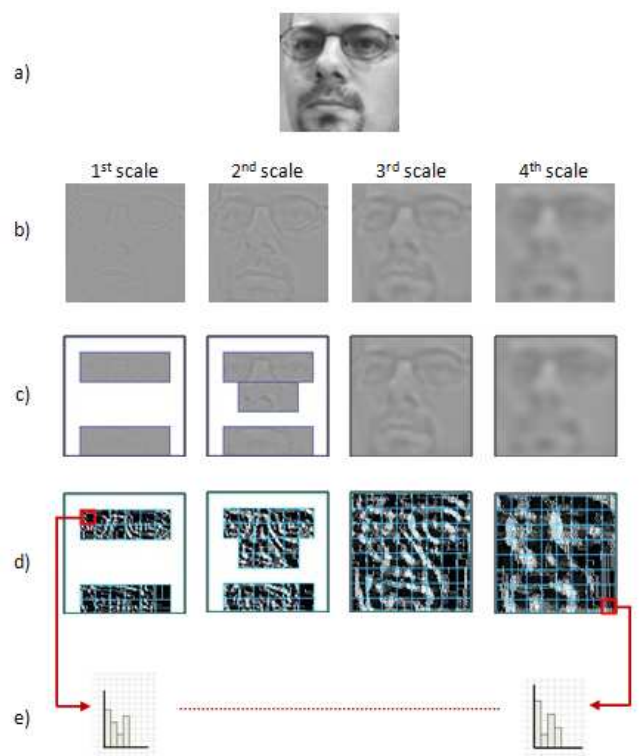

Figure 5: Different steps of proposed method. a) Initial image b) Result of DOG application into four scales c) ROIs of each scale d) Application of LBP and subdivision into blocks e) Histogram computation on each block and concatenation of all histograms to get the final vector.

\subsubsection{Matching by weighting ROIs and scales}

The second application of the bio-inspired approach in the proposed method consists in considering the importance of each ROI and each scale in the recognition task realized by observers in Schyns et al. work (Schyns, 2002). To this end, weighting coefficients were introduced in the Chisquare formula given by Equation 4:

$$
\chi_{\text {weight }}^{2}\left(\mathrm{H}_{1}, \mathrm{H}_{2}\right)=\sum_{s} w_{s} \sum_{r} w_{r} \chi^{2}\left(\mathrm{H}_{1}, \mathrm{H}_{2}\right)
$$

where $w_{r}$ and $w_{s}$ are, respectively, the weighting coefficient of each region $r$ and of each scale $s$.

The assignment of weights was guided by the findings in (Schyns, 2002). These findings state that, according to regions, the eyes were the most important followed by the mouth than the nose; while according to scale, the third scale was the most discriminative one, followed narrowly by the fourth one; the first and second scales having less influence on the recognition task. 


\section{RESULTS AND DISCUSSION}

In this section, the influence of a non exhaustive list of parameters related to the proposed bio-inspired method is firstly presented. Then, a comparison with other algorithms performed on IV ${ }^{2}$ project is brought. The results are reported with the Equal Error Rate percentage (EER).

\subsection{Choice of LBP variant}

A first set of experiments has been conducted to see which LBP variant was the most discriminant in the face authentication task. Three extensions of LBP operator were tested on the four $\mathrm{IV}^{2}$ experiments, besides the original LBP. Results in table 1 are in favor of $\mathrm{LBP}^{\mathrm{U} 2}$ as stated in many works in the literature (Huang, 2011).

Table 1: Comparison between LBP variants. Application on 4-scale DOG filtered ROIs divided into $16 \times 16$ blocks.

\begin{tabular}{lcccc}
\hline LBP variant & Exp1 & Exp2 & Exp3 & Exp4 \\
\hline$L B P$ & $5.1( \pm 0.7)$ & $16.2( \pm 1.2)$ & $21.4( \pm 1.6)$ & $16.9( \pm 1.5)$ \\
$L B P^{R I}$ & $5.9( \pm 0.8)$ & $18.1( \pm 1.3)$ & $22.7( \pm 1.6)$ & $16.6( \pm 1.4)$ \\
$L B P^{U 2}$ & $4.9( \pm 0.7)$ & $\mathbf{1 6 . 0}( \pm 1.2)$ & $20.8( \pm 1.6)$ & $16.5( \pm 1.4)$ \\
$L B P^{R I U 2}$ & $5.9( \pm 0.8)$ & $18.5( \pm 1.3)$ & $23.0( \pm 1.7)$ & $16.9( \pm 1.5)$ \\
\hline
\end{tabular}

\subsection{Choice of block size and ROIs}

A second set of tests was carried out to see whether it was better to keep the filtered images at their entirety as an input for $\mathrm{LBP}^{\mathrm{U} 2}$ histograms computation or to split it into blocks. Different block sizes were tested in this experimentation. Only the three best configurations are shown in Table 2, i.e.: entire image, $16 \times 16$ blocks and $32 \times 32$ blocks. It can be seen, that splitting the filtered images into blocks improves the results sensitively.

Another set of tests was run to prove the importance of the bio-inspired approach based on ROIs rather than the totality of blocks on each scale.

Table 2: Results of five configurations of $\mathrm{LBP}^{\mathrm{U} 2}$ application, including block size variation and ROIs selection.

\begin{tabular}{|c|c|c|c|c|c|}
\hline & Bloc size & Exp1 & Exp2 & Exp3 & Exp4 \\
\hline LBP image & - & $7.0( \pm 0.8)$ & $\begin{array}{l}27.3 \\
( \pm 1.5)\end{array}$ & $\begin{array}{l}31.6 \\
( \pm 1.8)\end{array}$ & $\begin{array}{l}29.2 \\
( \pm 1.8)\end{array}$ \\
\hline \multirow{2}{*}{ LBP all blocks } & 16 & $4.9( \pm 0.7)$ & $\begin{array}{r}17.4 \\
( \pm 1.3)\end{array}$ & $\begin{array}{l}24.8 \\
( \pm 1.7)\end{array}$ & $\begin{array}{r}15.0 \\
( \pm 1.4)\end{array}$ \\
\hline & 32 & $4.7( \pm 0.7)$ & $\begin{array}{r}19.7 \\
( \pm 1.3)\end{array}$ & $\begin{array}{l}25.8 \\
( \pm 1.7)\end{array}$ & $\begin{array}{c}16.3 \\
( \pm 1.4)\end{array}$ \\
\hline \multirow{2}{*}{$\begin{array}{l}\text { LBP blocks } \\
\text { on ROI }\end{array}$} & 16 & $4.9( \pm 0.7)$ & $\begin{array}{r}16.0 \\
( \pm 1.2)\end{array}$ & $\begin{array}{l}20.8 \\
( \pm 1.6)\end{array}$ & $\begin{array}{r}16.5 \\
( \pm 1.4)\end{array}$ \\
\hline & 32 & $4.0( \pm 0.6)$ & $\begin{array}{r}15.8 \\
( \pm 1.2)\end{array}$ & $\begin{array}{r}17.3 \\
( \pm 1.5)\end{array}$ & $\begin{array}{l}17.1 \\
( \pm 1.5)\end{array}$ \\
\hline
\end{tabular}

Results of the last two configurations in Table 2 show that significant improvements were provided by using only blocks of ROIs. The gain in terms of EER goes up to $8.5 \%$ in case of Experiment 3 which deals with quality variation, when using $32 \times 32$ blocks.

\subsection{Improvements by weighting ROIs and scales}

Four strategies of weighting in the Chi-square distance were tested. Weights were attributed empirically with reference to Schyns et al. studies. The kept weighting coefficients are as follows:

- According to regions: 0.6 for the eyes, 0.2 for the mouth, 0.12 for the nose and 0.08 for the rest of regions.

- According to scales: 0.05 for first scale, 0.1 for second scale, 0.45 for third scale and 0.4 for fourth scale.

Table 3: Influence of the weighting strategy.

\begin{tabular}{lcccc}
\hline $\begin{array}{l}\text { Weighting } \\
\text { strategy }\end{array}$ & Exp1 & Exp2 & Exp3 & Exp4 \\
\hline $\begin{array}{l}\text { No weighting } \\
\text { Weighting }\end{array}$ & $4.0( \pm 0.6)$ & $15.8( \pm 1.5)$ & $17.3( \pm 1.5)$ & $17.1( \pm 1.5)$ \\
$\begin{array}{l}\text { scales } \\
\text { Weighting ROI }\end{array}$ & $4.0( \pm 0.6)$ & $16.1( \pm 1.2)$ & $18.1( \pm 1.4)$ & $17.6( \pm 1.5)$ \\
$\begin{array}{l}\text { Weighting } \\
\text { scales }+ \text { ROI }\end{array}$ & $\mathbf{3 . 7}( \pm 0.6)$ & $15.6( \pm 1.2)$ & $15.7( \pm 1.4)$ & $\mathbf{1 6 . 6}( \pm 1.4)$ \\
\hline
\end{tabular}

Table 3 shows that weighting both scales and regions achieved the best results for almost all the experiments, especially for Experiment 3 (quality variation) where enhancement reaches $4.5 \%$ of EER when compared to unweighted strategy.

To summarize, the optimum parameters of the proposed bio-inspired method are to filter the normalized $128 \times 128$ face images by a 4 -scale DOG. Then, compute $\mathrm{LBP}^{\mathrm{U} 2}$ histograms on each $32 \times 32$ block of the Regions Of Interest and concatenate them into a single feature vector. Afterward, perform matching using Chi-square distance weighting both scales and regions.

\subsection{Comparison with IV $^{2}$ evaluation campaign}

To evaluate the efficiency of the proposed algorithm, comparison with five other algorithms using the same $\mathrm{IV}^{2}$ database and protocol is presented in Table 4 (Mellakh, 2009). According to these results, proposed algorithm provides the best results in Experiment1 including small expression variation. It occupies the second place in Experiments 2 and 3 involving illumination and quality variations; and it is 
placed third when faced to multisession variation in Experiment 4.

It can be seen that proposed method outperforms conventional algorithms, i.e. PCA1, PCA2 and LDA, in almost experiments. Besides, when compared to modular PCA, that makes also use of Regions Of Interest, proposed algorithm performs better in all Experiments.

Table 4: Comparative results between proposed algorithm (green) and $\mathrm{IV}^{2}$ first evaluation campaign ones (black).

$\begin{array}{llcccc} & \text { Algorithms } & \text { Exp1 } & \text { Exp2 } & \text { Exp3 } & \text { Exp4 } \\ & \text { PCA1 } & 6.7( \pm 0.8) & 20.7( \pm 1.3) & 20.1( \pm 1.6) & 22.2( \pm 1.6) \\ & \text { PCA2 } & 7.3( \pm 0.8) & 21.6( \pm 1.4) & 13.6( \pm 1.4) & 16.3( \pm 1.4) \\ \text { Previous } & \text { mod PCA } & 5.3( \pm 0.7) & 20.7( \pm 1.4) & 19.5( \pm 1.6) & 20.5( \pm 1.5) \\ \text { Tests IV2 } & \text { LDA } & \mathbf{3 . 7}( \pm 0.6) & 22.5( \pm 1.4) & 21.7( \pm 1.7) & 19.7( \pm 1.5) \\ & \text { LDA/ Gabor } & 4.2( \pm 0.6) & \mathbf{1 2 . 0}( \pm 1.1) & \mathbf{8 . 3}( \pm 1.1) & \mathbf{1 1 . 3}( \pm 1.2) \\ & \text { Bio-inspired } & \mathbf{3 . 7}( \pm 0.6) & 15.2( \pm 1.2) & 12.8( \pm 1.3) & 16.7( \pm 1.4) \\ \begin{array}{l}\text { Proposed } \\ \text { method }\end{array} & \text { LBP } & & & & \end{array}$

On the other hand, it is true that both bio-inspired LBP and LDA/Gabor are multiscale and based on combining conventional. But it has to be underlined that, unlike the bio-inspired LBP algorithm, which does not include any pretreatment for the face images nor any pretreatment phase, the LDA/Gabor algorithm performs an anisotropic smoothing on images, before features extraction, which proved to be very efficient face to variabilities such as illumination, quality and multisessions. Also it includes a learning phase to get the projection space (Fisherfaces) used afterwards in the test phase. That explains why LDA/Gabor achieves the best results for Experiments 2, 3 and 4.

\section{CONCLUSION AND FUTURE WORK}

Through this work, a bio-inspired approach based on psychovisual studies has been introduced for 2Dface authentication. The approach combines $\mathrm{LBP}^{\mathrm{U} 2}$ with multiscale DOGs. It has been proved that considering only relevant regions and weighting both regions and scales brought sensitive improvements. Proposed method showed to be robust not only in controlled environment but also face to illumination and quality variations.

Future works are intended to investigate pretreatment before feature extraction. Further research would consider a learning stage to enhance performance.

\section{REFERENCES}

Belhumeur P.N., Hespanha J.P. and Kriegman D.J., 1997. Eigenfaces vs fisherfaces: Recognition using class specific linear projection. In IEEE Trans. on PAMI, 19(7):711-720.

Gosselin F. and Schyns P.G., 2001. Bubbles: a technique to reveal the use of information in recognition tasks. In Vision Research, Elsevier. 41:2261-2271.

Huang D., Shan C., Ardabilian M. and Wang Y., 2011. Local Binary Patterns and Its Application to Facial Image Analysis: A Survey. In IEEE Trans. On Systems, Man, and Cybernetics, 41(6):765-781.

Li S. Z. and Jain A. K., 2005. Handbook of Face Recognition. Springer.

Mäenpää T., Ojala T., Pietik"ainen M. \& Soriano M., 2000. Robust texture classification by subsets of local binary patterns. In Proc. 15th Inter. Conf. on Pattern Recognition, 3: 947-950.

Mellakh A., Chaari A.., Guerfi S. \& all., 2009. 2D Face Recognition in the $\mathrm{IV}^{2}$ Evaluation Campaign. In Conf. on ACIVS, Bordeaux, France.

Ojala T., Pietikäinen M., and Mäenpää T., 2002. Multiresolution gray-scale and rotation invariant texture classification with local binary patterns, In IEEE Trans. on PAMI, 24(7), 971-987.

Petrovska-Delacretaz D., Lelandais S., Colineau J. \& all., 2008. The $\mathrm{IV}^{2}$ Multimodal Biometric Database (Including Iris, 2D, 3D, Stereoscopic, and Talking Face Data), and the IV2- 2007 Evaluation Campaign. In 2nd IEEE Inter. Conf. on Biometrics: Theory, Applications and Systems (BTAS), USA.

Pietikäinen M. and Ojala T., 1999. Nonparametric texture analysis with simple spatial operators. In Proc. on Texture Analysis in Machine Vision, 3-12. Finland.

Pietikäinen M., Ojala T. and Xu Z., 2000. Rotationinvariant texture classification using feature distributions. In Pattern Recognition 33: 43-52.

Rodieck R. W. and Stone J., 1965. Analysis of receptive fields of cat retinal ganglion cells. In J. Neurophysiol. 28:833-849.

Schor C. M., Wood I., 1983. Disparity range for local stereopsis as a function of luminance spatial frequencies. In Vision Research 23, 1649-1654.

Schyns P.G., Bonnar L. and Gosselin F., 2002. SHOW ME THE FEATURES! Understanding Recognition from the Use of Visual Information. In Psychological Science. 13(5):402-409.

Sinha P., Balas B., Ostrovsky Y. and Russell R., 2006. Face Recognition by humans: Nineteen results all computer vision researchers should know about. In Proceedings of the IEEE, 94(11):1948-1962.

Su Y., Shan S.G., Chen X.L. and Gao W., 2009. Hierarchical ensemble of global and local classifers for face recognition. In Journal of IEEE Trans. On Image Processing, 18(8): 1885-1896.

Zhang X. and Jia Y., 2005. Face Recognition Based on Steerable Feature and Random Subspace LDA. In Int. workshop on Analysis and Modeling of Faces and Gestures (AMFG), 3723(2):170-183, Beijing, China. 